\title{
Trinta anos da criação do Centro de Apoio Educacional e Psicológico da Faculdade de Medicina de Ribeirão Preto - contexto histórico e realizações dos primeiros tempos
}

\author{
Thirtieth anniversary of the Center for Educational and Psychological \\ Support of the Ribeirão Preto Medical School:establishment and early years \\ Maria de Lourdes Veronese Rodrigues ${ }^{1}$ (D) , Josimara Magro Fernandez de Souza², Marcia Baumann di Stasio ${ }^{3}$, \\ Maria de Fátima Aveiro Colares ${ }^{4}$ (D), Ana Raquel Lucato Cianflone ${ }^{5}$ (D)
}

\begin{abstract}
RESUMO
O artigo tem por objetivo historiar o contexto da criação e o desenvolvimento do Centro de Apoio Educacional e Psicológico (CAEP) da FMRP-USP em seus anos iniciais. São destacados aspectos estruturais, como: constituição da Equipe Técnica, Apoio Administrativo e Grupo de Consultores; infraestrutura e relações institucionais do CAEP com colegiados e a Administração da Unidade. Também é descrito o contexto da elaboração do Regimento Interno do CAEP, com ênfase na apresentação dos princípios que nortearam as atividades desenvolvidas por este Centro.
\end{abstract}

Palavras-chave: Educação Médica; Psicologia Educacional; Assistência em Saúde Mental.

\begin{abstract}
The article aims to describe the context of the establishment and development of the Center for Educational and Psychological Support (CAEP) of FMRP-USP, in its early years. Structural aspects are highlighted, such as the structure of the Technical Team, Administrative Support and Group of Consultants, and infrastructure and institutional relations of CAEP with the ruling council and the FMRP Administration. It is also described the context of the elaboration of the CAEP Internal Regulation, with emphasis on the presentation of the principles that guide the activities carried out by the center.
\end{abstract}

Keywords: Education, Medical; Psychology, Educational; Mental Health Assistance.

1 Professora Titular Aposentada do Departamento de Oftalmologia, Otorrinolaringologia e Cirurgia de Cabeça e Pescoço, Faculdade de Medicina de Ribeirão Preto, Universidade de São Paulo (FMRP-USP). Coordenadora do CAEP em 2011. Consultora Sênior do CAEP desde 2017.

2 Membro Efetivo da Sociedade de Psicanálise de Ribeirão Preto (IPI). Psicanalista. Membro da Equipe Técnica do NAPP/CAEP (Psicóloga); $1990-1994$.

3 Auxiliar Administrativa do CAEP, desde 2006, FMRP-USP.

4 Uni-FACEP Centro Universitário Municipal de Franca. Coordenadora dos Cursos de Psicologia e de Medicina. Membro da Equipe Técnica do CAEP (Psicóloga); 1996-2019.

5 Professora Doutora do Departamento de Psicologia, Faculdade de Filosofia Ciências e Letras de Ribeirão Preto, Universidade de São Paulo (FFCLRP-USP). Membro da Equipe Técnica do NAPP/CAEP (Pedagoga); 1989-2001.

$\checkmark$ Maria de Lourdes Veronese Rodrigues. Av. Bandeirantes, 3900 - $12^{\circ}$ andar do Hospital das Clínicas - Monte Alegre. CEP: $14049-900$. Ribeirão Preto (SP), Brasil. mdlvrodr@fmrp.usp.br | Recebido em: 20/10/2020 | Aprovado em: 21/10/2020 
Na década de 1980 ocorreram importantes mudanças sociais, políticas, culturais, científicas e tecnológicas, que marcaram a história da humanidade, com impacto na Educação Médica ${ }^{1-6}$. Nessa época, somaram-se aos fatores que, desde o início do século $X X$ impuseram necessidades de mudança na formação de médicos, os novos métodos de atenção à saúde (preconizados pela Conferência de Alma Ata); o aumento da qualidade dos cuidados ambulatoriais; a diminuição do tempo de hospitalização; o desafio do surgimento de novas doenças; as pressões médico-legais; as dificuldades financeiras e as diretrizes da Conferência de Edinburgh ${ }^{7-13}$. No Brasil, outro fator importante foi a criação do Sistema Único de Saúde - SUS ${ }^{14}$, que modificou consideravelmente a assistência à saúde ${ }^{14}$, demandando modificações no perfil dos médicos a serem formados.

Assim, além das inovações anteriormente introduzidas, entre as quais se destacavam a ênfase na geração de conhecimentos, a criação de Hospitais-Escola e a valorização do Internato e da Residência Médica ${ }^{11,15-23}$, no final dos anos 1980 estavam vigentes, em diferentes Faculdades de Medicina: - a expansão dos cenários de ensino, incluindo a atenção primária, a experiência clínica precoce e a integração docente-assistencial em equipes multiprofissionais; - o ensino da Medicina de Família; - o aumento da participação dos alunos no processo de aprendizado, incluindo o estímulo à aquisição de habilidades para gerenciar a própria educação permanente; - a disponibilização de tempo livre para aquisição de cultura geral e para estudos não supervisionados; - as atividades de tutoria e mentoria; - a introdução de novas técnicas de avaliação; - a criação de disciplinas eletivas; - novos modelos de estruturas curriculares, como o aprendizado centrado em problemas e a organização por ciclos da vida; - a informatização e utilização de novas formas de comunicação; - a criação de laboratórios de simulação; - o investimento no desenvolvimento do raciocínio clínico; - e a criação de centros de educação médica, que visavam o apoio educacional e pedagógico aos alunos e a formação didática e pedagógica dos docentes, que foram assumindo múltiplos papéis ${ }^{4,10,11,24-38}$.

A Faculdade de Medicina de Ribeirão Preto (FMRP), desde sua implantação, teve a preocupação com a formação de seus professores na área de ensino ${ }^{39}$, sendo que alguns dos fundadores dos Departamentos, antes de implantá-los, visitavam Escolas Médicas do exterior, com financiamento da Fundação Rockefeller ${ }^{11,40,41}$. Esta política continuou a ser exercida de forma esporádica, ainda assim resultando no envolvimento de vários docentes com questões importantes do processo de ensino-aprendizagem ${ }^{11,39}$.

Desta forma, nos anos 1980, entre outros assuntos, estavam na pauta da Comissão de Graduação (CG) da FMRP a aprovação dos objetivos terminais do Curso de Graduação em Medicina; o início do delineamento de importante reforma curricular, com ampliação do Internato; e a criação de órgão de apoio psicopedagógico para os alunos e para formação pedagógica dos professores ${ }^{11}$. Com exceção do aprimoramento dos docentes, que só foi viabilizado de forma abrangente com a implantação, em 2017, do Centro de Desenvolvimento Docente para o Ensino - $\mathrm{CDDE}^{42}$, na gestão da Diretora Margaret de Castro, os outros projetos se concretizaram imediatamente ou a curto prazo.

Os primeiros registros da preocupação da FMRP com aspectos emocionais e de saúde mental dos estudantes datam dos anos $1960^{43}$. Mas foi na década de 1980, quando a CG foi presidida por um docente psiquiatra (muito envolvido com o ensino e com aspectos emocionais do corpo discente), que as discussões sobre o tema foram ampliadas. Com apoio do então Diretor, Prof. Dr. Dalmo de Souza Amorim, o projeto de implantação de um setor de apoio psicopedagógico, subordinado administrativamente à Diretoria e funcionalmente à CG, foi aprovado pela Congregação da Unidade em abril de $1988^{44,46}$. Com o suporte financeiro da Reitoria da USP, em 1989 foi contratada uma Pedagoga que, inicialmente, assessorou a Comissão de Graduação, e, no ano seguinte, a contratação de uma Psicóloga propiciou a criação do Núcleo de Apoio Psicopedagógico da FMRP (NAPP) - atual Centro de Apoio Educacional e Psicológico (CAEP) ${ }^{43-45}$.

A primeira equipe do NAPP foi composta pela Pedagoga Ana Raquel Lucato Cianflone e pela Psicóloga Josimara Magro Fernandez de Souza, que na fase inicial tiveram o apoio administrativo da Secretária da Comissão de Graduação, Elisabeth Dovicchi Magrini. Quando, ainda em 1990, o NAPP teve sede própria (um espaço adaptado, com problemas de infraestrutura e pouca privacidade para os 
atendimentos), no prédio denominado "Anfiteatros Novos", foi designada como Técnica Administrativa a Sra. Aparecida dos Santos Eloy ${ }^{46}$.

Inicialmente, o NAPP foi coordenado pelos sucessivos Presidentes da Comissão de Graduação Profs. Drs. Cláudio Roberto Carvalho Rodrigues (1990-1991), José Antunes Rodrigues (1991-1993) e Wiliam Alves do Prado (19931994). Em 1994, foi designado pela Comissão de Graduação para coordenar o NAPP o Prof. Dr. José Fernando de Castro Figueiredo ${ }^{46}$. Já nos primeiros tempos do CAEP, o Coordenador passou a ser eleito pelo Conselho Consultivo, sendo que o Professor Figueiredo foi reconduzido até 2001, quando solicitou sua substituição.

Os objetivos do NAPP foram bem definidos: - apoio psicopedagógico aos alunos dos Cursos de Medicina e Ciências Biológicas; - realização de estudos e investigações para a caracterização psicopedagógica da FMRP para colher subsídios para o desenvolvimento de projetos pertinentes; e documentar todo o processo, para servir de base à continuidade do serviço e das pesquisas ${ }^{43,45,46}$. No entanto, segundo Cianflone, Figueiredo e Colares ${ }^{43}$, houve a expectativa de que o NAPP, além de fornecer assistência psicológica aos alunos, resolvesse também "situações de impasse", que incluíam quadros psicóticos graves, tentativas de suicídio e outros problemas que ocorriam e eram preocupantes. Com o amadurecimento das discussões e com argumentos da equipe técnica, houve ajustamento às possibilidades reais do serviço, ficando entendido que a responsabilidade de prover assistência ampla seria institucional ${ }^{43,45,46}$.

Além de estabelecer comunicação com os alunos dos dois cursos que existiam na FMRP, uma das primeiras preocupações do NAPP foi entrar em contato com os serviços similares existentes no Brasil Escola Paulista de Medicina, Universidade Federal do Rio de Janeiro e Faculdade de Medicina da USP ${ }^{45,46}$, solicitando material bibliográfico, relacionado à acoIhimento e orientação psicopedagógica a estudantes, e realizando algumas visitas técnicas ${ }^{46}$.

O NAPP atendia demandas da Diretoria, como às referentes a pareceres sobre o Programa de Bolsa Trabalho da USP, e assessorava a Comissão de Graduação tanto em aspectos acadêmicos quanto em problemas - por exemplo em intercorrências decorrentes de distúrbios em eventos como o trote aos calouros e o ritual de finalização da disciplina de Patologia, chamado "Adeus Básico" (era comum haver excessos por parte de alguns alunos, que acabavam em vandalismos ou alguma violência entre eles), colaborava na organização e desenvolvimento da "Semana de Recepção aos Calouros", e na implantação do Grupo PET (Capes) e conduzia projetos de investigação. Além disso, tanto a Pedagoga quanto a Psicóloga participavam de eventos científicos das respectivas áreas de conhecimento ${ }^{46}$.

Constam nos arquivos do NAPP/CAEP ${ }^{46}$ que no seu primeiro ano de existência foram desenvolvidas as seguintes atividades: "- Estudos a respeito da população discente e sua realidade; - Atendimentos à demanda espontânea de orientação psicológica e orientação pedagógica; - Elaboração e viabilização do programa de recepção aos alunos ingressantes; - Análise da situação acadêmica dos alunos que solicitam matrícula com superposição de horário; - Coordenação da aplicação de questionários de avaliação de disciplinas e docentes, respondidos pelos alunos, referentes ao diagnóstico do Ensino de Graduação, solicitado pelo Conselho de Graduação; - Planejamento e início da coleta de dados de estudo prospectivo envolvendo a Faculdade de Economia e Administração e a FUVEST, sobre dados dos alunos; - Relatório à Assistência Financeira; -Encaminhamento, à Diretoria, de relatório circunstanciado sobre leituras extracurriculares dos alunos de graduação; elaboração de artigo para publicação; e - Assessoria à $C G$, na elaboração de avaliação de disciplinas e docentes, aplicado do $1^{\circ}$ ao $6^{\circ}$ ano, no período de matrícula de 1991". As procuras espontâneas eram atendidas pela Psicóloga em técnica de psicoterapia breve, sendo que foram realizadas algumas avaliações psicológicas e orientação de pais e professores em situações mais graves.

No início de outubro de 1991, a equipe técnica do NAPP solicitou ao Presidente da CG que fosse criado um Conselho Consultor que participasse de discussões e decisões junto com o NAPP e que tivesse os objetivos de: "- Elaboração de Regimento Interno para o NAPP, - Discussão e definição de uma conduta, a nível institucional, para casos que apresentem problemas psiquiátricos graves; e - discussão a respeito das características psicopedagógicas da instituição, com vistas à proposição de atividades de caráter preventivo junto aos alunos"46. 
Essa solicitação foi aprovada pela CG, com base em parecer emitido pelo Prof. Dr. Carlos Eli Piccinato, que recomendou que o Grupo de Consultores fosse composto por um Professor de Psiquiatria e um Professor de Psicologia (indicados pelo Departamento); um Representante Docente da Comissão de Graduação; e pelo Psicólogo e Pedagogo do NAPP. Em fevereiro de 1992, foi realizada primeira reunião do grupo de Docentes Consultores do Núcleo de Apoio Psicopedagógico, composto pelos Profs. Drs. Cláudio Roberto Carvalho Rodrigues, Maria Beatriz Martins Linhares e Afonso Dinis Costa Passos e, pela Psicóloga Josimara Magro Fernandez de Souza e pela Pedagoga Ana Raquel Lucato Cianflone. Foram sugeridas à equipe o planejamento e desenvolvimento das seguintes atividades: -Diagnóstico precoce de alunos com dificuldades; -Assistência psicopedagógica a esses alunos; - Orientação a familiares de estudantes e a docentes; - Encaminhamento de casos, quando necessário; Pesquisa; -Promoção de atividades de caráter preventivo; - Recepção e acompanhamento dos alunos ingressantes; e- Assessoria à CG quanto a questões psicopedagógicas ${ }^{46}$. Essas atividades permitiram que o NAPP, gradualmente, assumisse seu papel institucional, conforme proposto em seus objetivos, sem função disciplinadora. Também propiciou que o NAPP representasse um espaço confiável e seguro junto à população discente para questões relacionadas à Saúde Mental.

No ano de 1992 o NAPP continuou a exercer e ampliou as atividades que estavam sendo desenvolvidas, com destaque para a inclusão de visita dos calouros ao Hospital das Clínicas e realização do "Ciclo de Palestras relacionadas à Saúde Mental do Universitário", de agosto a novembro, com a participação de docente da FMRP e de convidados de outras Unidades da USP.

Ainda em 1992, a Pedagoga do NAPP solicitou adaptação de horários para ingressar no Programa de Pós-Graduação em Saúde Mental. A aprovação dessa solicitação foi o marco inicial da política até hoje estimulada pelo CAEP, de investimento na formação profissional de sua equipe técnica.

Em 1993, a Semana de Recepção aos Calouros foi enriquecida com várias palestras e a versão definitiva do Regulamento do NAPP, elaborado com o apoio dos Docentes Consultores, foi encaminhado à CG. Na mesma época o NAPP passou a ter conta bancária própria e autonomia para gerenciamento dos recursos. Além disso, o NAPP colaborou com a organização e realização do "FORUM CARL para avaliação do ensino de graduação"; elaborou (em colaboração com membro do Grupo de Docentes Consultores) estudo retrospectivo sobre atendimentos de estudantes de medicina em ambulatório de clínica psiquiátrica do Hospital das Clínicas da FMRP e outro sobre características de ensino-aprendizado na FMRP; e promoveu a Mesa Redonda "A questão da Morte na Relação Médico-Paciente"46-48. Ainda em 1993, outro artigo de autoria da Pedagoga do CAEP foi publicado ${ }^{49}$.

Na mesma época, a equipe técnica do NAPP foi convidada a participar do "Grupo de Trabalho para a Avaliação Terminal de Competências dos Graduandos em Medicina", importante instrumento para a verificação da eficácia da reforma curricular recentemente implantada ${ }^{50,51}$. A participação neste Grupo de Trabalho possibilitou consolidar o papel de assessoria do NAPP à CG, estabelecido nos objetivos iniciais de sua criação.

A mesma equipe continuou a trabalhar em parceria em múltiplas atividades até junho de 1994, quando a Psicóloga Josimara Magro Fernandez de Souza entrou e licença maternidade e, em dezembro do mesmo ano, solicitou desligamento. No entanto, participou ativamente da elaboração do Relatório do NAPP, 1990-1994, apreciado pelo Grupo de Consultores e aprovado pela CG em dezembro deste $\mathrm{ano}^{46}$. Somente em 1996 sua vaga foi preenchida por Maria de Fátima Aveiro Colares, que logo assumiu o seu papel no Grupo de Trabalho para a Avaliação46,50.

Com o término do primeiro mandato do Grupo de Consultores do NAPP, em 1994, passaram a fazer parte dele Carmen Cinira dos Santos Martins e José Fernando de Castro Figueiredo. Maria Beatriz Martins Linhares foi reconduzida por mais um mandato. Em 1995, decidiu-se ampliar a esse Grupo e passaram a fazer parte o Prof. Dr. Sérgio Britto Garcia e o representante discente Omar Mamud Sales ${ }^{46}$.

As atividades regulares do NAPP em 1994 e 1995 e a participação no Grupo de Trabalho para a Avaliação propiciaram a apresentação de trabalhos em eventos nacionais e internacionais ${ }^{52-59}$, incluindo 
o "Annual Meeting of the Association for Medical Education in Europe - AMEE". Esta última participação deu início à tradição da presença de pelo menos um docente da FMRP em cada uma das vinte e cinCo "AMEE conferences" seguintes.

Ainda em 1994, foi observado que, de acordo com a legislação da Universidade de São Paulo (USP) ${ }^{46}$, o termo "Núcleo" era juridicamente incorreto para as características do NAPP. Assim, foi elaborado, com a colaboração da Assistente para Assuntos Acadêmicos, Sra. Cleide Filipini e da Analista de Recursos Humanos da Prefeitura do Campus-USP Ribeirão Preto, Sra. Lícia Barcelos de Souza, Projeto de transformação do NAPP em Centro de Apoio Educacional e Psicológico (CAEP), com novo Regulamento Interno. A apreciação minuciosa do Relatório NAPP 1990-1994 muito contribuiu para a definição dos papéis do CAEP, como passou a ser denominado a partir de 1996, sendo que o então Coordenador do NAPP, Prof. Dr. José Fernando de Castro Figueiredo, continuou a coordenar esse Centro ${ }^{46}$.

Ao longo de três décadas, com o trabalho árduo das sucessivas equipes técnicas e do pessoal administrativo, com a coordenação de docentes comprometidos e com o apoio dos consultores e das Diretorias da FMRP, o CAEP está consolidado, mas sempre buscando o aprimoramento. Está continuamente atento às tendências atuais do Ensino das Ciências da Saúde ${ }^{60}$, inclusive respondendo positivamente aos desafios da pandemia Covid-19, com mudanças pertinentes.

Atualmente é coordenado pelos Profs. Drs. Victor Evangelista de Faria Ferraz e Maria Paula Panúncio Pinto, que estão conduzindo a continuação da história de sucesso iniciada pelos pioneiros do Núcleo de Apoio Psicopedagógico da Faculdade de Medicina de Ribeirão Preto da Universidade de São Paulo.

\section{AGRADECIMENTOS}

Aos membros da Equipe Técnica da Revista Medicina (Ribeirão Preto) Marcos Vinicius Prospero e Edinê Barros Motta, por viabilizarem o acesso a documentos não digitalizados e pela grande disponibilidade.

\section{REFERÊNCIAS}

1. Amorim DS. As implicações do avanço científico-tecnológico na definição do perfil do médico. Medicina (Ribeirão Preto) 1989; 22(3/4):144-149.

2. Association of American Medical Colleges. Annual Report 1986-1987. J Med Educ. 1988; 63(3): 231-270.

3. Ferreira JR. A formação de recursos humanos para a Medicina no ano 2000. Rev Bras Educ Med. 1983; 7(2): 93-100.

4. Fordham CC. Changing Medical Education in new schools. N Engl J Med. 1980; 301(13): 719-720.

5. Jonas JS, Etzel SI, Barzansky B. Undergraduate Medical Education. JAMA 1989; 262(8): 1011-1019.

6. Pereira JC. Ensino Médico: Sociedade e Educação Médica. Medicina (Ribeirão Preto)1980; 12(3/4):17-20.

7. Reynolds RC. Medical Education 1980s. J Med Educ. 1980; 55(8):718-719.

8. Muller S. The Medical School in the University. JAMA 1984; 252(11):1455-1457.

9. Barbosa FS. Solilóquio da Educação: em apoio às transformações na Escola Medica Brasileira. Rev Bras Educ Med. 1986; 10(3):129-130.

10. Kendall PL, Reader GG. Inovations in Medical Education of the 1950s contrasted with those of the 1970s and 1980s. J Health Soc Behav. 1988; 29(4):279-293.

11. Rodrigues MLV. Inovações no Ensino Médico e outras mudanças: aspectos históricos e na Faculdade de Medicina de Ribeirão Preto. Medicina (Ribeirão Preto) 2002; 35(3):231-235.

12. Martins AM. Editorial. Rev Bras Educ Med. 2008; 32(1):5-6.

13. Gual A, Nuñez-Cortés JM, Palés-Argullós J, Oriol-Bosch A. FEM 2013; 16 (4): 186-189.

14. Yazlle Rocha JS, Caccia-Bava MCGG. O SUS, esse desconhecido. In: Rodrigues MLV, Kara José N. Perfil epidemiológico das principais causas de cegueira no Brasil. Rio de Janeiro: Cultura Médica, 2012. p. 87-92.

15. Mello HK. Hospital Escola - o problema visto pelo professor de Medicina. Rev Bras Educ Med. 1977; 1(1):3-5.

16. Yazlle Rocha JS. O Hospital Universitário e as tendências atuais da Educação Médica. Rev Bras Educ Med. 1979; 3(2):41-45.

17. Silva PCT. Internato. Rev Bras Educ Med. 1981; 5(2):117-123.

18. Gonçalves EL. O Hospital de Ensino: ontem hoje e amanhã. Rev Bras Educ Med. 1984; 8(3): 188-192.

19. Rosa AR. Internato: atualidade e perspectivas. Rev Bras Educ Med. 1985; 9(11):30-36.

20. Bradford WD, Schofield JF. Study of required clerkships in Internal Medicine in US and Canadian Medical Schools. J Med Educ. 1986; 61(3):157-162. 
21. Dias del Castillo E. El sistema de enseñanza de las especialidades médicas. Gaceta Médica de México 1987; 123(3/4):39-57.

22. Mauro JEM, Nogueira AR. Aspectos do Ensino Médico em São Paulo:1912-50. In: Mauro JEM, Nogueira AR. FMRP-USP. Primeiros tempos, através dos documentos e pela voz de seus construtores. FUNPEC:Ribeirão Preto; 2004. p.3-10.

23. Rodrigues MLV, Marchini JS, Salgado HC, Carlotti Jr CG. A consolidação da FMRP-USP/marcos históricos das primeiras décadas. In: Rodrigues MLV, Marchini JS, Salgado HC, Carlotti Jr CG. Faculdade de Medicina de Ribeirão Preto- USP. Primeiras Décadas. FUNPEC: Ribeirão Preto; 2018. p. 67-82.

24. Barrows HS, Tamblym RM. Problem-based learning: an approach to medical education. New York: Springer; 1980.

25. Pontes JPL. Ensino Médico no Brasil: evolução, perspectivas e desafios. Rev Bras Educ Med. 1982; 6(2):104-116.

26. Ginsburg AD. Comparison of in training evaluation with tests of clinical ability in medical students. J Med Educ. 1985; 60(1):29-38.

27. Kuske TT, Fleming GA, Jarechy RK, Levine JH, Lewis LA. Curriculum change in the 1980s - a report of 40 southern US Medical Schools. JAMA 1985; 254(19): 2783-2786.

28. Luna R. Reorientación de la educación médica. Educ Med Salud. 1986; 466-472.

29. Sobral DT. Participação estudantil na melhoria da Educação Médica. Rev Bras Educ Med. 1986; 10(1): 19-22.

30. Vidal CA, Quiñones J. Integración docente-assistencial. Educ Med Salud. 1986; 20(1):1-25.

31. Crowley AE; Etzel SI, Petersen ES. Undergraduate Medical Education. JAMA 1987; 258(8):1013-1020.

32. Koch-Weser D. Community-based education of the health professions in Latin America and US. Am J Publ Health. 1987; 77(4): 412.

33. Mc Leod PJ. Faculty development practices in Canadian Medical Schools. CMAJ. 1987; 136(1): 709-712.

34. Brasil. Ministério da Educação/Secretaria de Educação Superior/Comissão de Especialistas em Ensino Médico. Ensino Médico-Bases e Diretrizes para sua reformulação. Brasilia: MEC; 1987.

35. Oliva Aldamiz H. La reforma de los planos de estúdio em las facultades de medicina españolas. Rev Clin Esp. $1987 ; 181(8): 445-52$.

36. Pallie W, Carr DH.The Mc Master Medical Education Philosophy in theory, practice and historical perspective. Med Teacher. 1987; 181(8):59-71.

37. Rodrigues MLV, Figueiredo JFC. Aprendizado centrado em problemas. Medicina (Ribeirão Preto) $1996 ; 29(4): 396-402$.

38. Rodrigues MLV. Investigação sobre inovações no Ensino Médico (Estágio em Escolas Médicas do México, Estados Unidos e Canadá- Programa USP/BID). Relatório Técnico. Ribeirão Preto: FMRP; 1990.
39. Rodrigues MLV, Rodrigues CRC. Formação de Recursos Humanos na área de Ensino Médico na Faculdade de Medicina de Ribeirão Preto. Educ Med Salud. 1992; 26(2):280-284.

40. Ferreira Santos RE. Memórias. Um cirurgião operando a própria vida. Ribeirão Preto: FUNPEC; 2002.p. 207-234.

41. Azeredo AP. Memorial das Atividades Educacionais. Ribeirão Preto: FUNPEC; 2015. p.33-35.

42. Centro de Desenvolvimento Docente para o Ensino/ Faculdade de Medicina de Ribeirão Preto. Ribeirão Preto (SP): Bollela VR; maio 2018 [set 2020; acesso em 30 set 2020]. http://cdde.fmrp.usp.br/.

43. Cianflone ARL, Figueiredo JFC, Colares MFA. O Centro de Apoio Educacional e Psicológico (CAEP) da Faculdade de Medicina de Ribeirão Preto (USP): História e perspectivas. Medicina (Ribeirão Preto) 2002; 35(3): 392-396.

44. Faculdade de Medicina de Ribeirão Preto. Of. ATAC.158090, 1990.

45. Fernandez JM, Cianflone ARL. O Núcleo de Apoio Psicopedagógico da Faculdade de Medicina de Ribeirão Preto - USP. Medicina (Ribeirão Preto) 1991; 24(2):122-127.

46. Núcleo de Apoio Psicopedagógico/Centro de Apoio Educacional e Psicológico - FMRP. Arquivo Documental 1990-1996.

47. Fernandez JM, Rodrigues CRC. Estudo retrospectivo de uma população de estudantes de medicina atendidos no ambulatório de clínica psiquiátrica do Hospital das Clínicas da Faculdade de Medicina de Ribeirão Preto. Medicina (Ribeirão Preto) 1993; 26(2): 258-269.

48. Cianflone ARL, Fernandez JM. Algumas características do ensino e aprendizado na Faculdade de Medicina de Ribeirão Preto - USP: um estudo junto aos alunos de graduação. Medicina (Ribeirão Preto)1993; 26(2): 228-236.

49. Cianflone ARL, Figueiredo MAC. A representação da medicina como profissão: considerações teóricas sobre conteúdos levantados junto a estudantes secundaristas e universitários. Medicina (Ribeirão Preto) 1993; 26(2): 237-245.

50. Troncon LEA, Cianflone ARL, Rodrigues MLV, Piccinato CE, Peres LC, Figueiredo JFC. Avaliação Terminal de competências dos graduandos em Medicina: Relato da experiência inicial na Faculdade de Medicina de Ribeirão Preto. Ribeirão Preto: Gráfica Canavacci; 1996.

51. Troncon LEA, Rodrigues MLV, Piccinato CE, Figueiredo JFC, Peres LC, Cianflone ARL.Overcoming difficulties in the introduction of a summative assessment of clinical competence in a Brazilian Medical School. In:Scherpbier AJJA, van der Vleuten CPM, Rethans JJ, van der Steeg AFW. Advances in Medical Education. Kluwer Academic: London; 1997. p. 197-199.

52. Troncon LEA, Rodrigues MLV, Piccinato CE, Figueiredo JFC, Peres LC, Cianflone ARL. Avaliação Terminal de competências clínicas dos graduandos - experiência inicial da Faculdade de Medicina de Ribeirão Preto - USP. Anais, XXIII Congresso Brasileiro de Educação Médica; 27-29 out 1995; Porto Alegre (RS). Rio de Janeiro (RJ): ABEM; 1995. p. 35. 
53. Peres LC, Troncon LEA, Rodrigues, MLV, Figueiredo JFC, Cianflone ARL.Dificuldades no desenvolvimento de avaliação terminal de competências clínicas de graduandos em Medicina. Anais, XXIII Congresso Brasileiro de Educação Médica; 27-29 out 1995; Porto Alegre (RS). Rio de Janeiro (RJ): ABEM; 1995. p. 38.

54. Cianflone ARL, Troncon, LEA, Rodrigues MLV, Piccinato CE, Peres LC, Figueiredo JFC . Evaluation of clinical competence assessment. Abstracts Book. Annual Meeting of the Association of Medical Education in Europe; 7-9 set 1995; Zaragoza (Espanha): AMEE/SEDEM; 1995. p. 97.

55. Study of medical profession social representation aiming psychopedagogic counselling for medicine students. Abstracts Book. Annual Meeting of the Association of Medical Education in Europe; 7-9 set 1995; Zaragoza (Espanha): AMEE/SEDEM; 1995. p.35.

56. Figueiredo JFC, Troncon LEA, Cianflone ARL, Peres LC, Piccinato CE . Performance of students during the last years of medical School assessed by a multiple choice test over two consecutive years. Abstracts Book. Annual Meeting of the Association of Medical Education in Europe; 7-9 set 1995; Zaragoza (Espanha): AMEE/ SEDEM; 1995. p.98.
57. Troncon,LEA $A_{+}$Figueiredo $\mathrm{JFC}_{+}$Rodrigues $\mathrm{MLV}_{+}$Piccinato $\mathrm{CE}_{+}$ Peres LC, Cianflone ARL, Colares, MFA . Extra-curricular research activities and academic performance of medical students. Abstracts Book. Annual Meeting of the Association of Medical Education in Europe; 30 Ago-2 Set1997; Viena (Áustria). Dundee (Escócia): AMEE; 1995. p. 29.

58. Cianflone ARL, Peres LC ; Figueiredo JFC, Troncon LEA, Rodrigues MLV, Piccinato CE . Meta-evaluation in clinical competence assessment (OSCE) of Brazilian Medical School. Abstracts Book. Annual Meeting of the Association of Medical Education in Europe; 1-4 set 1996; Copenhagen (Dinamarca). Dundee (Escócia): AMEE; 1995. p.70.

59. Cianflone ARL, Figueiredo, JFC ; Rodrigues, MLV ; Colares, MFA . What are the characteristics of a good physician? Replies by medical students from a Brazilian Medical School. Abstracts Book. Annual Meeting of the Association of Medical Education in Europe; 30 Ago -2 Set 1997; Viena (Áustria). Dundee (Escócia): AMEE; 1995. p. 74.

60. Association for Medical Education in Europe. Dundee (Escócia): AMEE; ago 2020 [set 2020; acesso em 29 set 2020]. https://amee.org/conferences/amee-pastconferences/amee-2020. 